\title{
PARTICIPACIÓN ELECTORAL EN LAS ELECCIONES PRIMARIAS EN URUGUAY
}

Daniel Buquet Rafael Piñeiro

\begin{abstract}
Resumen
Uruguay es el único país hasta ahora en el que todos los partidos han realizado elecciones primarias abiertas y simultáneas para seleccionar su candidato presidencial. Como el voto en las primarias es voluntario, los efectos de esta norma sobre los resultados electorales están dados por las características del electorado que participa en ellas. A partir de datos de encuesta se analizan los factores que inciden sobre la participación y sus efectos sobre el tipo de candidatos presidenciales que tienden a ser electos. Los resultados encontrados confirman las presunciones teóricas mostrando que los electores más educados, que simpatizan con los partidos, y tienen posiciones ideológicas más extremas tienen mayor propensión a participar. En consecuencia, es esperable que los pre-candidatos que se ubican en posiciones más radicales resulten beneficiados por el uso de primarias.
\end{abstract}

Palabras clave: Participación Electoral; Partidos; Elecciones Primarias; Sistema Electoral; Uruguay.

\begin{abstract}
So far Uruguay is the only country in the world in which parties carried out open and simultaneous primary elections in order to select their presidential candidates. The effects of employing primaries on the election results are determined by the particular characteristics of the electorate who voluntarily participate in them. From survey data, we analyze the factors affecting participation in such instances and their effects on the type of presidential candidates tend to be elected. The results show that in primary elections vote citizens more educated, more interested in politics, identified with political parties and with more extreme ideological positions. As a result we observe that through primaries, candidates away from the median voter are more likely to be winners than with DSV system.
\end{abstract}

Keywords: Electoral Participation; Parties; Primary Elections; Electoral Systems; Uruguay.

\section{Introducción}

Uruguay es el único país en el que los partidos están obligados a realizar elecciones primarias abiertas y simultáneas para seleccionar su candidato presidencial $^{1}$. Como el voto en las primarias es voluntario, los

\footnotetext{
${ }^{1}$ Recientemente Argentina estableció una legislación similar, que se puso en práctica por primera vez en agosto de 2011, pero ninguno de los partidos registrados presentó competencia por la
} 


\section{DOSSIÊ OPINIÃO PÚBLICA, ELEIÇÕES E PARTICIPAÇÃO POLÍTICA NAS DEMOCRACIAS CONTEMPORÂNEAS}

efectos de esta norma sobre los resultados electorales están dados por las características del electorado que participa en ellas. En este artículo se utilizan datos de encuesta para analizar los factores que inciden sobre la participación en las elecciones primarias y sus efectos sobre el tipo de candidatos presidenciales que tienden a ser electos.

La participación electoral en Uruguay no ha sido un fenómeno muy estudiado. Los altos niveles de participación (rondan el $90 \%$ ), han tendido a ser explicados en función de la obligatoriedad de voto en las elecciones nacionales y departamentales, y como consecuencia de una cultura cívica desarrollada donde los partidos son los principales agentes de movilización política. El abstencionismo no parece haber sido un problema político para Uruguay y por lo tanto tampoco se ha transformado en un asunto de preocupación académica.

El interés de la ciencia política por el fenómeno de la participación electoral se basa en los impactos que sobre la calidad democrática puedan tener las bajas tasas de participación e involucramiento en la política. En este sentido, altos niveles de desafección pueden indicar también bajos niveles de competencia y -por lo tanto- poco control sobre los agentes políticos por parte de los electores. Como señalam Adserà, Boix y Payne (2003) si no existe accountability disminuye el estímulo que genera la "conexión electoral" a conductas políticas deseables. Por otro lado, como también indica Lijphart (1997), los sesgos en las características políticas y sociales entre quienes participan y quienes no lo hacen, pueden determinar que los agentes políticos se sientan responsables ante un electorado reducido que termina teniendo preferencias políticas muy diferentes a las del total de los electores.

En las elecciones primarias que se utilizan en Uruguay para seleccionar a los candidatos presidenciales de cada partido, el voto es voluntario. En teoría, los electores tienen la oportunidad de participar en esas primarias para definir el candidato de su partido. En consecuencia, la decisión no la toman los dirigentes del partido, sus afiliados, o sus votantes, sino aquellos que por la razón que sea concurren a votar en la elección primaria. Si bien se puede argumentar que este es un mecanismo más democrático, abierto y representativo que la selección por parte de un comité partidario, su grado de representativo está en función de quiénes participan en la elección primaria. En otras palabras, el grado de representatividad de la selección depende de cuánto las preferencias de esos votantes se parezcan a las del electorado del partido $o$ a las de sus afiliados (si se piensa que unos $u$ otros son quienes deben seleccionar al candidato). Lo que parece claro es que los electores que terminan decidiendo el candidato son muchos más que los afiliados de los partidos y muchos menos que sus votantes potenciales.

En este trabajo, en primer lugar, se repasa brevemente la discusión teórica acerca de los factores que inciden en la participación electoral y en

candidatura presidencial. 
particular aquellos que corresponden a elecciones primarias. Luego se describen las características del proceso de elecciones primarias en Uruguay. Finalmente se presenta un modelo que analiza la probabilidad que tienen los votantes de participar en las primarias en función de datos de la Encuesta Panel del Taller de Procesos Electorales 2009 del Instituto de Ciencia Política de la Facultad de Ciencias Sociales de la Universidad de la República y se analizan las posibles distorsiones que acarrea este sistema de selección.

\section{Por qué vota la gente}

Blais (2006) sostiene que a pesar de que la ciencia política ha dedicado muchos esfuerzos en comprender qué estimula y qué disuade la participación electoral, aún se conoce poco, ya que los hallazgos no son contundentes. Sin embargo, Geys (2006) marca que la percepción de Blais no es del todo correcta. En su "meta-investigación" logra sistematizar un set de variables que muestran -a lo largo de diversos estudios agregados sobre la participación electoral- efectos similares. De cualquier forma, tanto uno como otro remarcan la importancia de avanzar en el análisis combinado de variables que inciden a nivel macro y micro.

Un punto interesante que establece Geys sobre el estudio de la participación electoral es que no existen variables omnipresentes en las diferentes investigaciones. Para él, esto responde a la falta de una teoría firme sobre en qué basar la selección de variables. A este respecto, la propuesta teórica de Downs (1957), asentada en la lógica de la elección racional, parece ser la más aceptada. Este autor sostiene que, dado que el votar implica un costo, los individuos pagarán ese precio si su voto puede generarle un beneficio, en otras palabras incidir en el resultado. En consecuencia, la participación aumenta cuando las elecciones se dirimen por pocos votos, y/o cuando las instituciones aumenten el costo de no votar.

La "teoría económica" sobre la participación suele ver al voto como una inversión. A esta visión se le puede contraponer otra -que también es económica- que supone que el voto es un bien de consumo (ANSOLABEHERE, FIGUEIREDO y SNYDER, 2002). Bajo esta línea de razonamiento no se vota en función del beneficio esperado como en el caso de una inversión, sino en función de maximizar la utilidad (como en el caso del consumo de bien). Esta utilidad puede estar asociada al placer de ganar, al placer de ver que mi opción política obtiene buenos resultados, etcétera. Si bien considerar al voto como un bien de consumo, se asemeja a la teoría del voto expresivo, tiene por detrás otras implicaciones y mantiene algunas de las propiedades de la teoría downsiana (sobre todo las asociadas al costo). Por ejemplo, ir a votar guardaría algún tipo de relación con el ingreso, ya que las personas de bajos ingresos preferirían consumir otro tipo de bienes más necesarios que votar. Pero más importante aún, también bajo esta teoría se puede pensar en situaciones de alienación o indiferencia cuando las posiciones ideológicas de 


\section{DOSSIÊ OPINIÃO PÚBLICA, ELEIÇÕES E PARTICIPAÇÃO POLÍTICA NAS DEMOCRACIAS CONTEMPORÂNEAS}

los partidos se alejan de las del votante, o se superponen haciéndolos indistinguibles. Para las elecciones primarias en Uruguay, la hipótesis de los costos asociados al registro de los votantes y a las sanciones (factores institucionales) no tiene relevancia en tanto todos, quienes concurren y quienes no lo hacen tienen los mismos costos. En cambio, sí es relevante cuán cerrada parece la contienda entre candidatos, ya que esto impacta sobre la posibilidad de que el voto sea decisivo.

Con respecto a este último punto, Cox y Munger (1989) proponen una alternativa al análisis de los costos del votante asociados a cuán decisivo es su voto. Para ellos, en las elecciones más ajustadas hay mayor participación porque las elites desarrollan mayores esfuerzos por movilizar a los votantes a fin de asegurar el resultado. Aldrich (1993), por su parte, sostiene que los políticos invierten más en competencias cerradas y que esa inversión se ve reflejada en mayores niveles de participación. Por lo tanto, para él la participación aumenta en escenarios de competencia parejos, aún si los votantes individualmente no perciben que su voto podría ser decisivo. En esta línea de razonamiento Pérez-Liñán (2001, p. 288), al analizar la participación electoral en América Latina, sostiene que: "[...] we may have been looking at the wrong level of the game. It is the state, parties, and other agents - rather than formal rules and procedures - who provide selective incentives for voters to turn out". En consecuencia, dados los estímulos que una elección pareja produce en el votante (o en las élites partidarias), sería esperable que en las primarias en Uruguay los simpatizantes de los partidos con competencias más cerradas tuvieran una mayor participación.

Desde otro enfoque, Hajnal y Lewis (2003) analizan la participación electoral en elecciones municipales en California. Ellos advierten que la participación en elecciones concurrentes, en las que se realizan referendos y en las que los representantes que se eligen tienen más posibilidades de desarrollar políticas, presentan mayores niveles de participación. Extrapolando su razonamiento a las elecciones primarias en Uruguay sería esperable una mayor participación de simpatizantes de partidos con mayores chances de acceder al gobierno y por lo tanto de incidir en las políticas.

Por otra parte, y asociada a las características particulares de las elecciones primarias, existe la idea (proveniente de la experiencia norteamericana) de que en este tipo de elecciones los electores que tienen posiciones ideológicas más radicales, son más educados y tienen mayores ingresos presentan mayor propensión a votar (KEY, 1956; RANNEY, 1972; KENNEY y RICE, 1985). Si esto es así, el votante mediano (aquel que define la mayoría) del electorado que participa en las primarias está más a la derecha o más a la izquierda - según se ubique ideológicamente el partido - que el del electorado total. En consecuencia, los partidos que realizan primarias abiertas corren el riesgo de terminar presentado candidatos menos competitivos - más alejados del centro - que los que seleccionan candidatos a través de decisiones de comités partidarios (a priori más motivados que los electores 
del partido que participan en las primarias a tomar decisiones orientadas estratégicamente a ganar las elecciones generales).

En América Latina las primarias para seleccionar candidatos presidenciales son excepcionales, sin embargo existe una tendencia al aumento en su utilización (CAREY y POLGA-HECIMOVICH, 2006; ALCÁNTARA SÁEZ, 2002). La investigación sobre este tema en el continente - aunque escasa - ha propiciado un interesante debate sobre los efectos de estos mecanismos de selección sobre los resultados electorales. Por un lado, están quienes tienden a refrendar los postulados teóricos emanados de los trabajos de American Politics y a verificar su aplicación en los casos latinoamericanos (COLOMER, 2003; BUQUET y CHASQUETTI, 2008). Por otro Carey y PolgaHecimovich (2006) han puesto en cuestión los efectos negativos de las primarias en América Latina.

Carey y Polga-Hecimovich (2006) sostienen que, al contrario de lo que se piensa para Estados Unidos, en América Latina la utilización de primarias para seleccionar candidatos presidenciales otorga una ventaja en la elección general. Su argumento se basa en que los electores en América Latina (continente en que los partidos son evaluados negativamente por los ciudadanos) ven a los que utilizan primarias como democráticos y transparentes. Adicionalmente, los electores no necesariamente seleccionan peores candidatos que los dirigentes partidarios, dado que pueden favorecer a aquellos con alto arraigo popular sin poder suficiente dentro de la estructura partidaria. Por último, las primarias pueden ser mecanismos eficientes para resolver conflictos sobre candidaturas, en particular en el caso de coaliciones - alianzas de carácter electoral. Sin embargo, los resultados empíricos presentados en el trabajo de estos autores - no son concluyentes respecto a la presencia de un "bonus" electoral - que sostienen existe - para los partidos que realizan primarias para seleccionar candidatos presidenciales. De cualquier forma, sí parecen descartar posibles efectos negativos.

Existen otras variables que suelen ser mencionadas por la literatura como significativas para explicar la participación, en particular en elecciones primarias. Kenney y Rice (1985) muestran cómo el nivel educativo, unido a las bajas barreras legales a la inscripción y a elecciones históricamente competitivas tienden a estimular la participación electoral en las elecciones primarias en los Estados Unidos. Suele señalarse también que la presión social y el hábito incide sobre la participación electoral. A este respecto, Gerber, Green y Shachar (2003) muestran cómo la propensión a votar aumenta en función de la participación en anteriores elecciones.

Por último, Campbell et al. (1960), y lo que usualmente se denomina escuela de Míchigan, subrayan la importancia de factores psicológicos en el comportamiento político. En particular, en su versión más tradicional, ponen acento sobre la socialización política como determinante de la identificación partidaria. Estos autores sostienen que: "[...] some of the dimension of attitude that are most helpful in accounting for turnout appear to have the 


\section{DOSSIÊ OPINIÃO PÚBLICA, ELEIÇÕES E PARTICIPAÇÃO POLÍTICA NAS DEMOCRACIAS CONTEMPORÂNEAS}

character of orientations to politics much more than they do the character of forces acting on a present decision" (CAMPBELL et al., 1960, p. 93). Para ellos la probabilidad de que una persona vote depende entonces de la intensidad de la preferencia partidaria e interactúa con la percepción de lo ajustado del resultado de la contienda electoral. En el caso del análisis del comportamiento electoral en las primarias, Geer (1988) señala que el interés en las elecciones primarias no está determinado por el status socioeconómico sino fundamentalmente por la identificación partidaria. Sostiene que no son los votantes más ricos los que votan en las primarias sino los que Campbell (1960) Ilama "core voters", los más cercanos al partido.

En función de las explicaciones teóricas respecto a la participación electoral, en particular en elecciones primarias, sería esperable que quienes participen en este tipo de instancias sean electores más educados, con posiciones ideológicas más extremas y que simpatizan con partidos que tienen competencias más parejas y cuentan con mayores chances de acceder al gobierno nacional.

\section{La interna de los partidos en la competencia presidencial en Uruguay}

La reforma electoral de 1997 obligó a los partidos a presentar una candidatura única para presidente y vicepresidente, y terminó con el doble voto simultáneo (DVS) para la elección presidencial (BUQUET y CHASQUETTI, 2008). El DVS permitía que los partidos presentaran más de una fórmula presidencial, de forma que los electores podían votar por un partido y luego seleccionar una de las fórmulas presidenciales que ese partido presentaba. Resultaba electa la fórmula más votada dentro del partido más votado. En la práctica, este sistema hacía que en un mismo acto electoral se realizaran elecciones primarias o internas y la elección presidencial.

La eliminación de un sistema tan particular como el DVS para la elección presidencial supuso la incorporación de un mecanismo no menos original para seleccionar en cada partido la candidatura única a presidente y vicepresidente, elecciones primarias abiertas y simultáneas para todos los partidos. La particularidad del nuevo sistema estriba en que los partidos están obligados a realizar estas elecciones de manera simultánea cuatro meses antes de la elección nacional ${ }^{2}$.

En las elecciones primarias resultan electos dentro de cada partido aquellos candidatos que obtengan la mayoría absoluta de los votos dentro de su partido, o un $40 \%$ y al menos 10 puntos porcentuales de diferencia con quien entre en segundo lugar. Si ningún candidato alcanza alguno de estos

\footnotetext{
${ }^{2}$ El artículo $8^{\circ}$ de la Ley 17.063 -que dicta normas relativas a las elecciones internas de los partidos- establece que: "Las elecciones internas referidas en la Disposición Transitoria W) de la Constitución de la República se realizarán en un único acto, con sufragio secreto y no obligatorio, en el que en una hoja de votación, identificada por el lema partidario, se expresará el voto por el candidato único del partido político a la Presidencia de la República [...]".
} 
dos requisitos, la nominación queda en manos de un colegio elector nacional elegido en la misma instancia.

Los promotores de la reforma señalaban que el sistema de DVS tendía a distorsionar las preferencias políticas de los electores. Este argumento se representa en la Figura 1. Allí se representa a dos partidos, el Partido de Izquierda que presenta dos candidatos $\left(\mathrm{I}_{1}\right.$ e $\left.\mathrm{I}_{2}\right)$, y el Partido de Derecha que presenta un único candidato (D). En el ejemplo, el candidato D del Partido de Derecha es el más votado individualmente $(48 \%)$, sin embargo quien resulta electo es el candidato $\mathrm{I}_{1}(32 \%)$ perteneciente al Partido de Izquierda, ya que este partido obtuvo el $52 \%$ de los votos al incluir el $20 \%$ de los votos correspondientes al candidato $\mathrm{I}_{2} \mathrm{y}$, por lo tanto, superar al Partido de Derecha. Nótese que el candidato $\mathrm{I}_{2}$ del Partido de Izquierda tiene una posición más cercana a $\mathrm{D}$ que a $\mathrm{I}_{1}$, lo que supone que si este último hubiera sido el candidato único de su partido y los electores se inclinaran a votar por quien le es más cercano en términos ideológicos, el resultado podría haber sido otro. Solo habría hecho falta que un poco más del $2 \%$ de los electores que votan por $\mathrm{I}_{2}$ cuando se utiliza el DVS, tuvieran como segunda preferencia a $\mathrm{D}$ de tal forma que este último triunfaría sobre el candidato $\mathrm{I}_{1}$ si fuera candidato único de su partido.

Si bien el diagnóstico puede discutirse, la solución a través de impedir las candidaturas múltiples elimina cualquier posibilidad de una eventual distorsión de las preferencias electorales. Sin embargo, la obligación de realizar internas o primarias abiertas y simultáneas para definir a los candidatos únicos no es una regla inocua y sus consecuencias dependen entre otros factores de los sesgos que presente el electorado que participa en ellas.

Fiqura 1 - Eiemplo de competencia electoral con DVS

Partido de Izquierda

(52\% del electorado)
Partido de Derecha

(48\% del electorado)

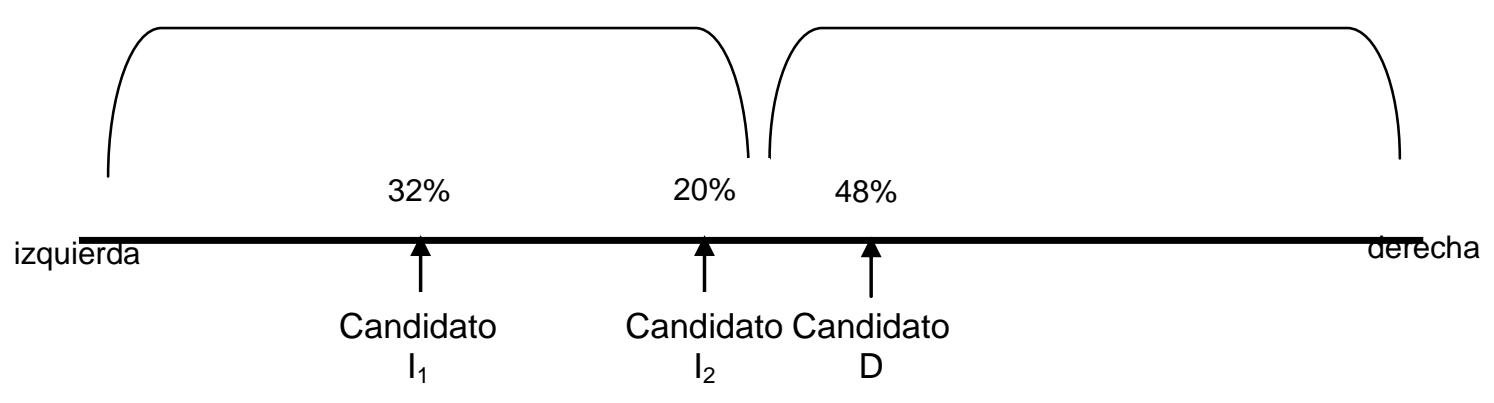

Fuente: Elaboración propia.

La experiencia acumulada en la utilización de primarias abiertas desde la elección presidencial de 1999, permite extraer algunas tendencias presentes a lo largo de la última década. En primer término las elecciones 


\section{DOSSIÊ OPINIÃO PÚBLICA, ELEIÇÕES E PARTICIPAÇÃO POLÍTICA NAS DEMOCRACIAS CONTEMPORÂNEAS}

primarias han estimulado su utilización efectiva para designar el candidato presidencial de los partidos principales ${ }^{3}$. Si bien esto puede resultar natural para los partidos tradicionales que siempre habían utilizado el DVS con esa finalidad, no es así en el caso del Frente Amplio (FA) que, al contrario, siempre criticó la multiplicidad de candidaturas presidenciales. La obligatoriedad de las elecciones internas y la evolución política del FA condujeron finalmente a que este partido también haya resuelto definir su candidato presidencial en una competencia interna abierta, en una decisión que parece no tener retorno mientras exista más de un aspirante a la nominación. Por otra parte, la naturaleza mayoritaria de la elección primaria (más allá de los umbrales establecidos) genera incentivos para que la competencia se concentre entre dos competidores principales. La evolución del número efectivo de candidatos (NEC), si bien se redujo para todos los partidos en 2004 con respecto a 1999, terminó ubicándose en valores aproximados a 2 para todos los partidos en 2009. Finalmente también puede apreciarse una regularidad estratégica: los partidos con internas competitivas entre dos candidatos principales tienden a armar su fórmula presidencial con el ganador como presidente y el segundo como vicepresidente (Batlle-Hierro en 1999, Lacalle-Larrañaga en 2009 y Mujica-Astori en 2009). Este fenómeno ocurrió en todos los casos en que la diferencia entre el primero y el segundo (DIF) fue inferior a quince puntos porcentuales y siempre que se utilizó resultó ganadora una fórmula con esas características. Evidentemente esta estrategia pretende compensar el efecto perjudicial que una reñida competencia interna pueda tener en el desempeño del partido en la elección general (ATKESON, 1998). Se intenta así evitar, por un lado, que las críticas realizadas por el perdedor de la primaria puedan ser utilizadas por los rivales $y$, por otro lado, que sus votantes deserten del partido como consecuencia de la derrota.

\footnotetext{
${ }^{3}$ A diferencia del caso argentino donde en 2011 ningún partido presentó competencia.
} 
Tabla 1 - Votación de los dos primeros candidatos, diferencia y número efectivo de candidatos

\begin{tabular}{l|c|c|c|c|c}
\hline & $\mathbf{1 9 9 9}$ & & $\mathbf{2 0 0 4}$ & & $\mathbf{2 0 0 9}$ \\
\hline Batlle & $54,92 \%$ & Stirling & $\mathbf{9 1 \%}$ & Bordaberry & $\mathbf{7 2 , 2 \%}$ \\
\hline Hierro & $44,14 \%$ & Iglesias & $\mathbf{7 \%}$ & Amorín & $14,7 \%$ \\
\hline DIF & $10,8 \%$ & DIF & $\mathbf{8 4 , 2 \%}$ & DIF & $57,5 \%$ \\
\hline NEC & 2,01 & NEC & 1,2 & NEC & 1,8 \\
\hline Lacalle & $48,3 \%$ & Lacalle & $34 \%$ & Lacalle & $57,1 \%$ \\
\hline Ramírez & $32,3 \%$ & Larrañaga & $66 \%$ & Larrañaga & $42,8 \%$ \\
\hline DIF & $16,0 \%$ & DIF & $33 \%$ & DIF & $14,3 \%$ \\
\hline NEC & 2,8 & NEC & 1,8 & NEC & 2,0 \\
\hline Vázquez & $82,4 \%$ & Vázquez & $100 \%$ & Mujica & $52,0 \%$ \\
\hline Astori & $17,6 \%$ & & & Astori & $39,7 \%$ \\
\hline DIF & $64,8 \%$ & DIF & $100 \%$ & DIF & $12,4 \%$ \\
\hline NEC & 1,4 & NEC & 1 & NEC & 2,3 \\
\hline Fuente: & & & & &
\end{tabular}

Fuente: Elaboración propia a partir de datos del Área de Política y Relaciones Internacionales del Banco de Datos de la Facultad de Ciencias Sociales de la Universidad de la República.

\section{Análisis de datos}

A partir de datos de la Encuesta Panel del Taller de Procesos Electorales 2009 del Instituto de Ciencia Política de la Facultad de Ciencias Sociales de la Universidad de la República ${ }^{4}$, intentaremos advertir posibles efectos de las internas abiertas sobre la selección de candidatos presidenciales en Uruguay. Una primera mirada de los resultados podría hacernos concluir rápidamente que los candidatos más extremos de los partidos fueron los favorecidos en las elecciones primarias. Sin embargo, esto no permite concluir que se deba al perfil de los votantes que participan en este tipo de elecciones, ya que podría tratarse simplemente de candidatos con mayor apoyo también en el conjunto del electorado.

A fin de observar las características de los electores participan en las elecciones primaras realizamos un modelo de regresión logística probit. Este

\footnotetext{
4 Se utiliza la base de datos de la segunda ola de una encuesta panel telefónica (558 casos entrevistados entre el 7 y el 15 de noviembre de 2009) realizada en el marco del taller Procesos Electorales de la Licenciatura en Ciencia Política de la Facultad de Ciencias Sociales, UdelaR. Se trata de una encuesta telefónica representativa de todo el país con muestreo probabilístico aleatorio polietápico por conglomerados con tres puntos de muestreo con afijación proporcional a la distribución de la población: nivel nacional, departamental y local. El marco de muestreo fue el listado de teléfonos georeferenciados, que tiene amplia cobertura en el Uruguay $(95 \%)$. El margen de error es de $+-4,1 \%$, con $95 \%$ de confianza para el total de la muestra y los márgenes de error de los efectos de cada variable en el modelo se reportan en la tabla correspondiente. Por tratarse de una encuesta posterior a las elecciones primarias, la variable dependiente se estima por recordación y no por intención de voto y los resultados están ponderados de acuerdo al resultado real de las primarias, por lo que se eliminan posibles sesgos por sobredeclaración de voto como ha ocurrido con las estimaciones de participación realizadas a partir de encuestas preelectorales (BOIDI y QUEIROLO, 2009).
} 


\section{DOSSIÊ OPINIÃO PÚBLICA, ELEIÇÕES E PARTICIPAÇÃO POLÍTICA NAS DEMOCRACIAS CONTEMPORÂNEAS}

modelo nos permitirá advertir los cambios en la probabilidad de votar en las primarias en función de la posición ideológica, controlando por otras variables como el nivel educativo, la simpatía partidaria, la edad, el lugar de residencia y el sexo del encuestado.

En función de la discusión teórica al respecto de los sesgos en la participación electoral, en particular en las elecciones primarias, esperamos que los votantes que ocupan posiciones ideológicas más extremas (tanto hacia la derecha como hacia la izquierda) también presenten una mayor probabilidad de ir a votar, controlando por variables que la literatura también señala como asociadas positivamente con la participación electoral. Es de esperar que la educación, la edad y la identificación partidaria presenten también efectos positivos sobre la probabilidad de ir a votar en tanto están asociados a mayor interés en la política y mayor propensión a ser movilizados por las campañas electorales.

Los resultados de este modelo son reportados en la Tabla 2 . Se observa que la probabilidad de votar en la interna o primaria en 2009 dependió de la edad, el nivel educativo, la posición ideológica y la identificación del encuestado con alguno de los tres principales partidos del sistema.

Para advertir el impacto de las diferentes variables en la probabilidad de participar en las elecciones primarias realizamos, a partir del modelo presentado, las simulaciones que aparecen en la Tabla 3 . En promedio, la probabilidad de votar en las elecciones primarias de un hombre de Montevideo, de 20 años, con secundaria terminada, que simpatiza con el FA y se ubica en el centro del espectro ideológico (posición 5), es de 0,28. Para un individuo de las mismas características, pero del Partido Nacional (PN), su probabilidad de participar en la interna es de 0,35. Si en lugar de 20 años tuvieran 60, la probabilidad sube en el caso del simpatizante del FA a 0,50 y en el del PN a 0,58. Con respecto al efecto de la posición ideológica del votante, vemos cómo estar en las posiciones más extremas (1 extrema izquierda y 9 extrema derecha) implica tener mayores probabilidades de ir a votar. Por ejemplo, esto supone para un simpatizante del FA de 40 años (de Montevideo y con secundaria completa) un aumento desde 0,39 - cuando se ubica en el centro - hasta 0,55 - si se ubica en la extrema izquierda. Para un nacionalista con las mismas características el incremento va de 0,46 - para los que se ubican en el centro - hasta un 0,62 - para los que se posicionan en la extrema derecha. 
Tabla 2 - Modelo Probit, variable dependiente voto en la interna ${ }^{5}$

\begin{tabular}{l|c|c}
\hline & Coeficientes & Errores estandarizados \\
\hline ideol & $0,1040^{*}$ & 0,0429 \\
\hline edad & $0,0150^{*}$ & 0,0032 \\
\hline educ & $0,1157^{*}$ & 0,0256 \\
\hline pn & $0,5787^{*}$ & 0,1537 \\
\hline $\mathrm{fa}$ & $0,3770^{*}$ & 0,1259 \\
\hline pc & $0,4274^{*}$ & 0,1823 \\
\hline mvd & $-0,2208^{*}$ & 0,1088 \\
\hline coxo & $-0,1382$ & 0,1043 \\
\hline constante & $-1,7225^{*}$ & 0,2669
\end{tabular}

Fuente: Elaboración propia a partir de datos de la Encuesta del Taller de Procesos Electorales de 2009 del Instituto de Ciencia Política FCS-UdelaR.

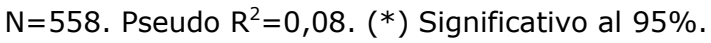

Con respecto al impacto del nivel educativo del votante sobre su probabilidad de ir a votar en la primaria, el modelo indica que un elector de 50 años, con educación primaria terminada, que vive en Montevideo, se autodefine como de centro (posición 5) y simpatiza con el FA, tiene una probabilidad participar en la interna en promedio de 0,24 . Mientras que un votante de las mismas características pero con estudios universitarios completos tiene una probabilidad de 0,54.

Por último, en el modelo también se pretendió advertir si los niveles de competitividad de las primarias en los diferentes partidos se asocian con la probabilidad de los simpatizantes de ir a votar. Un votante de 40 años, de Montevideo, con secundaria completa que se ubica en el centro (posición 5) y simpatiza con el PN tiene una probabilidad de votar de 0,46, mientras que un frenteamplista presenta un 0,39 y un simpatizante colorado 0,41. Sobre este punto el modelo no parece arrojar datos muy contundentes ya que si bien según los datos de opinión pública la interna del PN parecía la más cerrada (las principales encuestadoras daban en promedio una distancia de $16 \%$ entre los dos principales candidatos de este partido), la del FA aparecía como mucho más competitiva (21\%) que la del PC $(55 \%)^{6}$.

\footnotetext{
${ }^{5}$ La variable dependiente es voto en la interna, se codificó como 1 a quienes respondían en la encuesta que habían concurrido a votar en la primaria y como 0 a quienes reportaban no haberlo hecho (la pregunta fue formulada de la siguiente manera "Como usted sabe el pasado 28 de junio se realizaron las elecciones internas, en las que no era obligatorio ir a votar. ¿Usted votó en dichas elecciones?"). Asimismo, mvd es una variable binaria que adopta el valor de 1 cuando la persona vive en Montevideo y 0 cuando lo hace en el interior; sexo es una variable binaria donde el valor 1 se le asigna a los hombres y 0 a las mujeres; edad es la edad en años del encuestado; educ es una variable ordinal que va desde 1 (para encuestados sin instrucción) hasta 9 (individuos con universidad completa); ideol es el absoluto de la diferencia entre la posición ideológica reportada por el encuestado y el centro (distancia del centro); $p n, p c$ y fa son variables binarias que adoptan el valor de 1 cuando el encuestado indica ser simpatizante del PN, del PC o del FA respectivamente, y 0 cuando no lo son.

${ }^{6}$ Distancias promedio entre la intención de voto del primer y segundo candidato en la interna del
} 


\section{DOSSIÊ OPINIÃO PÚBLICA, ELEIÇÕES E PARTICIPAÇÃO POLÍTICA NAS DEMOCRACIAS CONTEMPORÂNEAS}

Estos resultados muestran que la posición ideológica, el nivel educativo y la edad (controlados por la simpatía por partido) tienen efectos significativos sobre la probabilidad de votar. En consecuencia, los candidatos preferidos de este tipo de electores se verían beneficiados por la realización de primarias abiertas (al menos en el caso de 2009).

Tabla 3 - Simulación de la probabilidad de votar en las elecciones primarias en función de simpatía partidaria, edad y posición ideológica*

\begin{tabular}{c|c|c|c|c}
\hline \multicolumn{1}{c}{ Partido } & Posición & $\mathbf{2 0}$ años & $\mathbf{4 0}$ años & $\mathbf{6 0}$ años \\
\hline \multirow{4}{*}{ Frente Amplio } & 1 & 0,43 & 0,55 & 0,66 \\
\cline { 2 - 5 } & 2 & 0,39 & 0,51 & 0,62 \\
\cline { 2 - 5 } & 3 & 0,35 & 0,47 & 0,59 \\
\cline { 2 - 5 } Partido Nacional & 4 & 0,31 & 0,42 & 0,54 \\
\cline { 2 - 5 } & 5 & 0,28 & 0,39 & 0,50 \\
\cline { 2 - 5 } & 5 & 0,35 & 0,46 & 0,58 \\
\cline { 2 - 5 } & 6 & 0,39 & 0,51 & 0,62 \\
\cline { 2 - 5 } & 7 & 0,43 & 0,54 & 0,66 \\
\cline { 2 - 5 } & 8 & 0,47 & 0,59 & 0,70 \\
\hline
\end{tabular}

${ }^{*}$ Simulación realizada a partir del modelo probit presentado en la Figura 1 con el paquete Zelig

(IMAI, KING y LAU, 2007 y 2008) del programa R. El resto de las variables del modelo fueron fijadas de la siguiente manera: $m v d=1$, $\operatorname{sex}=1, y$ educ $=7$ (hombre que vive en Montevideo con secundaria completa).

Fuente: Elaboración propia a partir de datos de la Encuesta del Taller de Procesos Electorales de 2009 del Instituto de Ciencia Política FCS-UdelaR.

\section{Sesgos en la distribución ideológica de los votantes en las primarias}

Si avanzamos un poco más y nos preocupamos por analizar la distribución ideológica del electorado en general y las de quienes participaron en las diferentes primarias podremos apreciar la dimensión del efecto de este sesgo sobre la definición de los candidatos en cada partido.

En el Gráfico 1 se pueden observar las distribuciones ideológicas del electorado, de los votantes de las primarias del PN y del FA, y de sus principales candidatos. En el caso de la competencia dentro del FA entre Danilo Astori y José Mujica, el primero no logra superar a Mujica entre los votantes ubicados en las distintas posiciones ideológicas. Sin embargo, la diferencia más pronunciada entre ambos candidatos parece concretarse entre los votantes de izquierda más radicales (posiciones 1 a 3 ). Es interesante observar cómo en la interna del FA vota más del $65 \%$ del electorado que se ubica en esas posiciones, mientras que solo algo más del $33 \%$ de quienes se ubican en la 4 y 5 (centro) lo hacen. Dentro del PN la importancia de los votantes con posiciones extremas parece aún más decisiva. Lacalle y de las empresas CIFRA, FACTUM, EQUIPOS MORI, RADAR e INTERCONSULT. 
Larrañaga aparecen virtualmente empatados entre los electores que se ubican en las posiciones 5 y 6 , mientras que el primero logra aventajar a Larrañaga en los votantes que están más a la derecha (posiciones 7 a 9). Al igual que lo que ocurre con los votantes de la primaria del FA, los de la del PN de centro son sólo poco más del $20 \%$ del total de votantes que se ubican en las posiciones 5 y 6 . Sin embargo son un $40 \%$ del total de los que se ubican más a la derecha (posiciones 7,8 y 9 ).

Estos datos, unidos a los del modelo estadístico presentado antes, parecen mostrar que efectivamente las primarias beneficiaron a candidatos ubicados en posiciones más extremas. Por un lado, un porcentaje mayor de quienes se encuentran en posiciones ideológicas distantes del centro tienden participar en las primarias ( $44 \%$ de quienes se ubican en la posición 5 vs. casi el $58 \%$ de los que se ubican en otras posiciones). Por otro lado, nótese que por estos votantes, fundamentalmente los que se ubican en las posición 5, los candidatos de uno y otro partido compiten también entre sí.

Si bien no sería correcto afirmar que los resultados electorales sin primarias abiertas hubieran sido otros, al menos en 2009, sí parece claro que en un sistema en que todos los electores participan, las competencias internas - tanto del Partido Nacional como del Frente Amplio - se hubieran resuelto de manera bastante más ajustada. Esto podría hacernos suponer que las primarias abiertas también, aunque tal vez en una forma diferente a la que según se decía podía hacerlo el DVS, tienda a desvirtuar las preferencias de los electores.

Gráfico 1 - Distribución ideológica del electorado general, de los votantes de la elección primaria del PN y del FA, y de sus principales candidatos

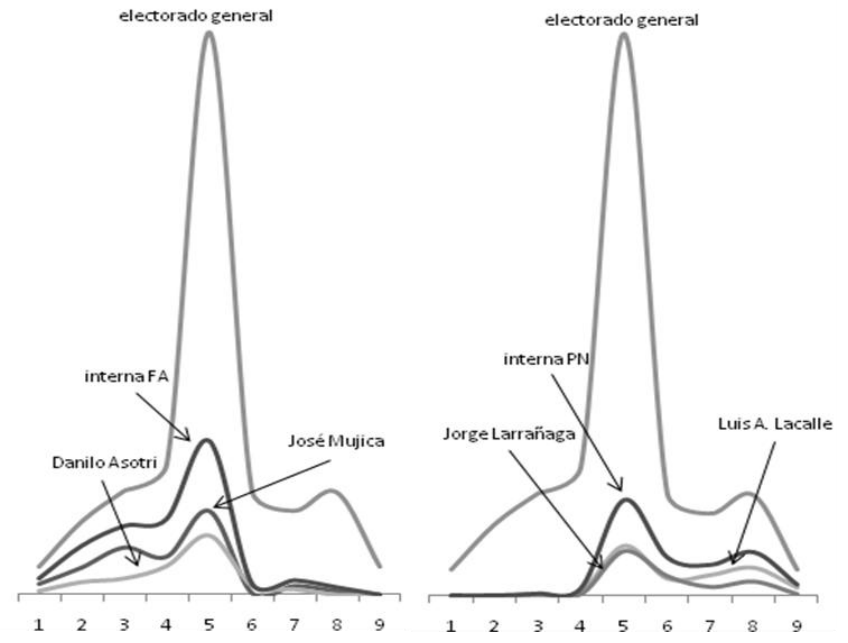

Fuente: Elaboración propia a partir de datos de la Encuesta del Taller de Procesos Electorales de 2009 del Instituto de Ciencia Política (FCS-UdelaR). 


\section{DOSSIÊ OPINIÃO PÚBLICA, ELEIÇÕES E PARTICIPAÇÃO POLÍTICA NAS DEMOCRACIAS CONTEMPORÂNEAS}

En Uruguay, la posición ideológica de los votantes que participan en las primarias tiene un sesgo hacia ubicaciones más extremas, refrendando los argumentos teóricos de quienes señalan que las primarias suelen beneficiar a candidatos más radicales. En la línea del razonamiento que realizan Carey y Polga-Hecimovich (2006) cuando todos los partidos seleccionan sus candidatos mediante elecciones primarias abiertas no es esperable que exista beneficio o perjuicio para alguno de ellos en la elección general. Sin embargo, se puede pensar que incluso en sistemas que obligan a la realización de primarias, un partido puede evadir este requisito evitando la competencia electoral, haciendo que sólo un candidato (elegido por el partido y supuestamente de centro) se presente a la elección. Si esta resolución no es conflictiva dentro del partido y está en línea con las preferencias de la mayoría de sus electores, no hay por qué pensar que los ciudadanos otorguen valor alguno a la definición del candidato a través de elecciones primarias competitivas. Por lo tanto, al menos en Uruguay, si existe un posible "bonus" estaría en función de que un partido logre definir el candidato entre dirigentes - que con visión estratégica - seleccionen candidatos moderados.

\section{Conclusión}

La reforma electoral de 1997 obligó a los partidos en Uruguay a seleccionar sus candidatos presidenciales en elecciones primarias, abiertas y simultáneas. Este sistema, que a priori se presenta como abierto y democrático, puede generar distorsiones en tanto quienes participan en estas elecciones tienen características diferentes a las de los potenciales votantes de los partidos y a las del electorado en su conjunto.

En este trabajo se han intentando identificar los factores que determinan la participación electoral en las elecciones primarias en Uruguay y los impactos que los sesgos en el electorado que participa podrían tener sobre la selección de los candidatos presidenciales. En función de datos de encuesta, los resultados del modelo estadístico presentado parecen refrendar buena parte de los postulados teóricos sobre la participación electoral y sobre la participación en elecciones primarias en particular.

La posición ideológica, el nivel educativo y la edad (controlados por la simpatía por partido) tienen efectos significativos sobre la probabilidad de votar. Los votantes con posiciones ideológicas más extremas, de mayor edad y nivel educativo son quienes muestran una mayor propensión a participar en estas elecciones. En cambio, la evidencia no es concluyente respecto a la hipótesis de que los votantes de los partidos que presentan competencias más cerradas tienen una mayor probabilidad de votar.

En consecuencia, los candidatos preferidos por este tipo de electores, aquellos que tienden a votar en primarias, se ven beneficiados por este mecanismo de selección. Este punto pone en cuestión la representatividad de 
esta forma de selección, ya que quienes seleccionan a los candidatos presidenciales de los partidos no son representativos del conjunto del electorado. En particular, el hecho de que los votantes con posiciones ideológicas más extremas muestren una mayor propensión a votar en las elecciones primarias presenta estímulos favorables a la polarización de la competencia y permite que resulten electos presidentes más alejados del votante mediano que cuando se utiliza otro método de selección.

Daniel Buquet es Doctor en Ciencia Política por la Facultad Latinoamericana de Ciencias Sociales (FLACSO-México), Profesor e Investigador del Instituto de Ciencia Política de la Facultad de Ciencias Sociales de la Universidad de la República de Uruguay, Investigador nivel II por la Agencia Nacional de Investigación e Innovación del gobierno de Uruguay.

E-mail: dbuquet@gmail.com

Rafael Piñeiro es candidato a doctor en Ciencia Política por la Pontificia Universidad Católica de Chile y docente e investigador del Instituto de Ciencia Política de la Facultad de Ciencias Sociales de la UdelaR de Uruguay, Presidente de la Asociación Uruguaya de Ciencia Política (AUCiP).

E-mail: rpineiro@uc.cl

\section{Referências}

ADSERÀ, Alícia; BOIX, Carles; PAYNE, Mark. Are You Being Served? Political Accountability and Quality of Government. The Journal of Law, Economics, and Organization, Oxford, v. 19, n. 2, p. 445-490, oct. 2003.

ALCÁNTARA SÁEZ, Manuel. Experimentos de democracia interna: Las primarias de partidos en América Latina. Kellogg Institute Working Paper, n. 293, apr. 2002. $49 \mathrm{f}$.

ALDRICH, John H. Rational choice and turnout Americal Journal of Political Science, Houston, v. 37. n. 1, p. 246-278, feb. 1993.

ANSOLABEHERE, Stephen; FIGUEIREDO, John de; SNYDER, James. Why Is There So Little Money in U.S. Politics? The Journal of Economic Perspectives, Pittsburgh, v. 17, n. 1, p. 256-271, jan. 2002.

ATKESON, Lonna Rae. Divisive Primaries and General Election Outcomes: Another Look at Presidential Campaigns. American Journal of Political Science, Houston, v. 42, n. 1, p. 256-271, jan. 1998.

BLAIS, André. What Affects Voter Turnout? Annual Review of Political Science, 
DOSSIÊ OPINIÃO PÚBLICA, ELEIÇÕES E PARTICIPAÇÃO POLÍTICA NAS DEMOCRACIAS CONTEMPORÂNEAS

Palo Alto, v. 9, p. 111-125, jun. 2006.

BOIDI, Fernanda; QUEIROLO, Rosario. La piedra en el zapato (de las encuestadoras): encuestas de opinión y elecciones internas 2009. Revista Uruguaya de Ciencia Política, Montevideo, v. 18, n. 1, p. 65-83, dic. 2009.

BUQUET, Daniel; CHASQUETTI, Daniel. Presidential Candidate Selection in Uruguay, 1942-2004. En: SIAVELIS, Peter M.; MORGENSTERN, Scott (Eds.). Pathways to Power. Political Recruitment and Candidate Selection in Latin America. Pennsylvania: Penn State University Press, 2008. p. 316-342.

CAMPBELL, Angus. Surge and decline: The study of electoral change. Public Opinion Quarterly, Oxford, v. 24, n. 3, p. 397-418, autumn 1960.

CAMPBELL, Angus et al. The American Voter. New York: Wiley, 1960.

CAREY, John M.; POLGA-HECIMOVICH, John. Primary Elections and Candidate Strength in Latin America. The Journal of Politics, Tucson, v. 68, n. 3, p. 530543, aug. 2006.

COLOMER, Josep. Las elecciones primarias presidenciales en América Latina y sus consecuencias políticas. En: CAVAROZZI, Marcelo; MEDINA, Juan Manuel Abal (Eds.). El asedio a la política: los partidos latinoamericanos en la era neoliberal. Buenos Aires: Altamira/Konrad Adenauer, 2003. p. 117-134.

COX, Gary W.; MUNGER, Michael C. Closeness, expenditures, and turnout in the 1982 U.S. House elections. American Political Science Review, Washington, v. 83, n. 1, p. 217-231, mar. 1989.

DOWNS, Anthony. An Economic Theory of Democracy. New York: Harper \& Brothers, 1957.

GEER, John G. Assessing the Representativeness of Electorates in Presidential Primaries. American Journal of Political Science, Houston, v. 32, n. 4, p. 929945, nov. 1988.

GERBER, Alan S.; GREEN, Donald P; SHACHAR, Ron. Voting May Be HabitForming: Evidence from a Randomized Field Experiment. American Journal of Political Sciencie, Houston, v. 47, n. 3, p. 540-550, jun. 2003.

GEYS, Benny. Explaining Voter Turnout: A Review of Aggregate-Level Research. Electoral Studies, Dallas, v. 25, n. 4, p. 637-663, dic. 2006.

HAJNAL, Zoltan L.; LEWIS, Paul G. Municipal Institutions and Voter Turnout in 
Local Elections. Urban Affairs Review, London, v. 38, n. 5, p. 645-668, may 2003.

- Toward A Common Framework for Statistical Analysis and Development. Journal of Computational and Graphical Statistics, Alexandria, v. 17, n. 4, p. 892-913, dic. 2008.

IMAI, Kosuke; KING, Gary; LAU, Olivia. Zelig: Everyone's Statistical Software. 2007. Disponible en: <http://GKing.harvard.edu/zelig>. Visitado el: 11 mayo 2010.

KENNEY, Patrick J.; RICE, Tom W. Voter Turnout in Presidential Primaries: A Cross-Sectional Examination. Political Behavior, Lexington, v. 7, n. 1, p. 101112, mar. 1985.

KEY, Valdimer Orlando. American state politics: An introduction. New York: Knopf, 1956.

LEY No 17.063. Dictanse Normas Relativas a las Elecciones Internas de los Partidos Políticos. Promulgada por el Poder Ejecutivo el $24 / 12 / 98$ y publicada en el Diario Oficial el 5/1/99.

LIJPHART, Arend. Unequal Participation: Democracy's Unresolved Dilemma. The American Political Science Review, Washington, v. 91, n. 1, p. 1-14, mar. 1997.

PÉREZ-LIÑÁN, Aníbal. Neoinstitutional accounts of voter turnout: moving beyond industrial democracies. Electoral Studies, Dallas, v. 20, n.2, p. 281297, jun. 2001.

RANNEY, Austin. Turnout and representation in American presidential elections. The American Political Science Review, Washington, v. 66, n. 1, p. 21-37, mar. 1972.

Texto recebido em 24/08/2011.

Aprovado em 07/12/2011. 